\title{
Chemical Composition of the Essential Oil of Hyptis Suaveolens
}

\author{
N. Peerzada \\ Faculty of Science, Northern Territory University, Casuarina, Darwin, Northern Territory, Australia 0909. Tel:61-8- \\ 89466360, Fax: 61-8-89466847, Email: N-Peerzada@ banks.ntu.edu.au
}

Received: 25 August 1997 / Accepted: 24 October 1997 / Published: 15 November 1997

\begin{abstract}
The chemical composition of Hyptis suaveolens was investigated and twenty three compounds were abundant enough to be identified by GC-MS.
\end{abstract}

Keywords: Hyptis suaveolens, Lamiaceae, chemical composition, cineole, caryophyllene.

\section{Introduction}

The Hyptis geneus (Lamiaceae) is composed of 400 species that mainly occur in tropical America [1-3]. Most of these plants are highly aromatic and also found in the other parts of the world; particularly as a weed in the wet tropic region of the Northern Territory, Australia [4-6]. The genus Hyptis is known to be used for traditional medicine for the treatment of various illness and has been found to possess significant pharmacological activity [712], including tumorigenic [13-16], antifertility [17,18] mycotoxic and phytotoxic activities [19]. H. suaveolens has recently been shown to possess insecticidal properties [20] as well as grain protectant from Cowpea weevil during storage [21]. The terpenes content of distilled volatile oils have been known to differ enormously due to different geographical locations and between different species of the same plant $(22,23)$. This genus possesses a diverse range of biological activities that led us to investigate the chemical composition of essential oil obtained by hydrodistillation from the leaves of Hyptis suaveolens Poit. The chemical constitutents of this specie was also compared with two other species, H. mutabilis [22] and H. emoryi [24].

\section{Results and Discussion}

The essential oil obtained after hydrodistillation of the leaves of $H$. suaveolens gave an average yield of $0.1 \%$. The main constitutents were 1, 8-cineole (32\%) and $\beta$ caryophyllene (29\%) Fig. 1. Enormous difference in the concentration levels of these two major components were found in the three species, $H$. suaveolens, $H$. mutabilis [22] and H. emoryi [24], as shown in the Table 1. This significant variation of the major components permit an easy differentiation between these three species. 


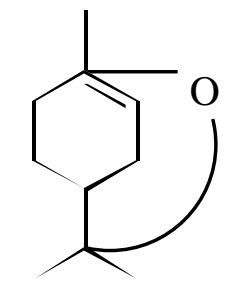

1,8-Cineole<smiles>CC1=CCC2C(C)CCC(C(C)C)C23CCC13</smiles>

$\alpha$-Copaene

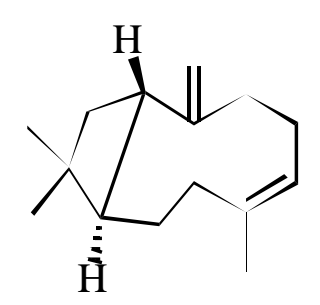

$\beta$-Caryophyllene<smiles>C=C(C)/C=C\C(C)C(C)C</smiles>

$\alpha$-Phellandrene

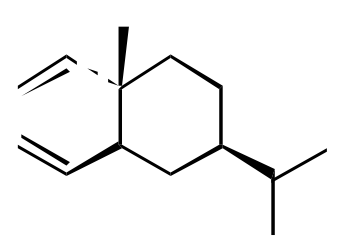

$\beta$-Elemene<smiles>C=CCc1ccc(O)c(OC)c1</smiles>

Eugenol

Fig. 1. Major components, 1,8- cineole and $\beta$-caryophyllene and others only found in Hyptis suaveolens 
Table 1. A comparison of chemical composition of the essential oils from Hyptis sp. $\mathrm{A}=H$. suaveolens; $\mathrm{B}=H$. emoryi [24]; $\mathrm{C}=H$. mutabilis [22].

\begin{tabular}{|c|c|c|c|}
\hline Component & $\mathrm{A}$ & B & $\mathrm{C}$ \\
\hline$\alpha$-Thujene & 0.3 & 7.0 & 1.80 \\
\hline$\alpha$-Pinene & 2.5 & 6.6 & 0.30 \\
\hline Camphene & 0.02 & 1.1 & - \\
\hline Sabinene & 3.9 & $\mathrm{~T}$ & 0.33 \\
\hline$\beta$-Pinene & 4.2 & 5.0 & 0.51 \\
\hline Myrcene & 0.6 & 1.8 & 1.85 \\
\hline$\alpha$-Phellandrene & 2.0 & $\mathrm{~T}$ & - \\
\hline$\alpha$-Terpinene & - & - & 0.74 \\
\hline p-Cymene & - & - & 15.14 \\
\hline Limonene & - & 5.6 & - \\
\hline$\beta$-Phellandrene & - & 0.8 & - \\
\hline 1, 8-Cineole & 32 & 6.9 & 5.67 \\
\hline cis- $\beta$-Ocimene & - & - & 0.74 \\
\hline$\gamma$-Terpinene & 0.7 & 0.1 & 1.93 \\
\hline$\alpha$-Terpinolene & 0.3 & $\mathrm{~T}$ & - \\
\hline Cimenenol & - & - & 0.33 \\
\hline Linalool & 0.06 & 1.3 & 1.77 \\
\hline Fenchol & 0.3 & - & - \\
\hline Camphor & - & 1.3 & - \\
\hline 4-Borneol & - & 11.9 & - \\
\hline 4-Terpinenol & 2.3 & 1.9 & 0.34 \\
\hline$\alpha$-Terpineol & 0.2 & - & 0.43 \\
\hline Carvone & - & 0.7 & - \\
\hline Neral & - & 2.0 & - \\
\hline Bornyl acetate & - & 0.6 & - \\
\hline Thymol & - & - & 7.85 \\
\hline Carvacrol & - & - & 0.35 \\
\hline$\delta$-Elemene & - & - & 1.44 \\
\hline Eugenol & 1.2 & - & - \\
\hline$\alpha$-Copaene & 1.8 & - & - \\
\hline$\beta$-Elemene & 1.0 & - & - \\
\hline$\beta$-Caryophyllene & 29 & 2.5 & 12.35 \\
\hline$\alpha$-Humulene & 1.6 & $\mathrm{~T}$ & 2.95 \\
\hline$\alpha$-Bergamotene & 2.0 & - & - \\
\hline Aromadendrene & 0.5 & - & 0.59 \\
\hline$\gamma$-cadinene & 0.1 & 6.7 & - \\
\hline$\delta$-cadinene & 0.5 & 1.4 & - \\
\hline
\end{tabular}

$\mathrm{T}=$ Trace 


\section{Materials and Methods}

\section{Plant Material}

Plant material was collected around Darwin and a vaucher specimen has been deposited at the Northern Territory University Herbarium.

\section{Distillation of the Essential Oils}

Fresh leaves were hydrodistilled for three hours to give pale yellow essential oil in $0.1 \%$ yield (w/w) [25]. The oil was dried over anhydrous sodium sulphate and analysed with GC-MS for identification of its components.

\section{GC-MS Analysis}

The component of the oils were identified by GC-MS analysis using a Varian Saturn GC-MS instrument. Capillary GLC was carried out by using at $25.0 \mathrm{~m}$ OV101 glass capillary column. The column was programmed at $90{ }^{\circ} \mathrm{C}$ for $10 \mathrm{~min}$ to $180^{\circ} \mathrm{C}$ at a rate of $2^{\circ} \mathrm{C} / \mathrm{min}$, then kept at $180^{\circ} \mathrm{C}$ for $30 \mathrm{~min}$. Helium was used as a carrier gas. Mass spectra were taken at $70 \mathrm{eV}$. The gc/ms data system contains the National Bureau of Standards (NBS) mass spectral library and Registry of Mass Spectral Data [26] were used to help verify the identity of individual components by mass spectral comparison. Identification of the separated components were also carried out by using authentic specimens and private library of essential oil constituents.

\section{References}

1. Willis, J. C. Dictionary of Flowering Plants and Ferns, (Rev. by Shaw, A. K.) Cambridge University Press; London. p 587, 1973.

2. Munz, P. A. A. California Flora; University of California Press; p 178, 1959.

3. Hutchinson, J.; Dalziel, J. M. Flora of West Tropical Africa, Crown Agents; London, p. 452. 1963.

4. Cameron, A. G. Aust. J. Exp. Agr. 1996, 36, 929-935.

5. Lonsdale, W. M.; Lane, A. M Biol. Conser. 1994, 69, 277-283.
6. Low, T. Bush Medicine: A Pharmacopoeia of Natural Remedies; Angus \& Robertson Publication, Sydney, NSW. Australia, 1990.

7. Nayak, V. G.; Guha, P. C. J. Indian Chem. Soc. 1952, 29, 183.

8. Kuhnt, M.; Probstle, A.; Rimpler, H.; Bauer, R.; Heinrich, M. Planta Medica 1995, 61, 227-232.

9. Kingston, D. G. I.; Rao, M. M.; Zucker, W. V. J. Nat. Prod. 1980, 42, 469.

10. Garg, S. K. Indian J. Med. Res. 1976, 64, 1133.

11. Chaudhery, R, R.; Hag, M. Bull. Med. Ethnobot. Res., 1980, 1, 408.

12. Iwu, M. M., Ezeugwu, C. O.; Okunji, C. O.; Sanson. D. R.; Tempesta, M. S. Int. J. Crude Drug Res. 1990 , 28,73 .

13. German, V. F. J. Pharm. Sci. 1971, 60, 649.

14. Sheth, K.; Jolad, S.; Wiedhopf, R.; Cole, J. R. J. Pharm. Sci. 1972, 61, 1819.

15. Kingston, D. G. I.; Zucker, W. V. J. Nat. Prod. 1980, $42,652$.

16. Saluja, S. K.; Santini, D. D. Indian Drugs 1981, 19, 127.

17. Saluja, S. K.; Santini, D. D.; Singh, J. D. Indian Drugs 1981, 19, 84.

18. Pandey, D. K.; Tripathi, N. N.; Dixit, S. N. Z. Pflanzenkr. Pflanzenschuiz 1982, 89, 344.

19. Porter, R. B. R.; Reese, P. B.; Williums, L. A. D.; Williums, D. J. Phytochem. 1995, 40, 735-738.

20. Singh, G.; Upadhyay, R. K. J. Sci. Ind. Res. 1993, 52, 676-683.

21. Fatope, M. O.; Nuhu, A. M.; Mann, A.; Takeda, Y. Inter. J. Pest Manag. 1995, 41, 84-86.

22. Luz, A. I. R.; Zoghbi, M. B.; Ramos, L. S.; Maia, J. G. S.; Da Silva, M. L. J. Nat. Prod. 1984, 47, 745.

23. Mukherjee, K.S.; Mukherjee, R. K.; Gosh, P. K. J. Nat. Prod. 1984, 47, 377.

24. Tanowitz, B. D.; Junak, S. A.; Smith, D. M. J. Nat. Prod. 1984, 47, 739.

25. Craveiro, A. A.; Matos, F. S. A.; Alencar, J. W. J. Chem. Educ. 1976, 53, 652.

26. Abrahamsson, S.; Stenhagen, E.; Mclafferty, F. W. Registry of Mass Spectral Data; Wiley, New York. 1974.

Sample Availability: Available from the authors. 